\title{
Sleep disturbances and epileptiform activity in a subpopulation of children with attention deficit hyperactivity disorder (ADHD): a literature review generating an hypothesis with implications for drug therapy
}

\author{
L.G. Miller and I.A. Kraft \\ Baylor College of Medicine, University of Texas School of Public Health, Houston, Texas, USA \\ Correspondence to: L.G. Miller, 5510 Greenbriar Road, Houston, TX 77005, USA
}

\begin{abstract}
The use of electroencephalography and sleep studies in attention deficit hyperactivity disorder (ADHD), and the associated behavioral disorders is reviewed. Based on the available literature, we propose a hypothesis indicating four subtypes of ADHD. The usefulness of EEGs and sleep laboratory indices in detecting a subgroup of patients with submaximal responses to methylphenidate is also discussed.
\end{abstract}

Keywords: Attention deficity hyperactivity disorder (ADHD) - Epileptiform activity - Sleep - Temperament

\section{INTRODUCTION}

Attention deficit hyperactivity disorder (ADHD) is a childhood behavior disorder characterized by inattentiveness, impulsivity, and overreactivity (APA, 1987). Historically, ADHD has been attributed to minimal brain dysfunction, and genetically-determined delayed cerebral maturation (Walker, 1950; Walker and Katy, 1958; Lahey et al., 1988). Neurophysiological abnormalities in ADHD have also been investigated. In a comprehensive review, Stevens et al. noted EEG abnormalities in $35-74 \%$ of patients with behavioral disorders compared with abnormal EEGs in 5-27\% of age-matched controls (Stevens et al., 1968). Thirty per cent of children with ADHD had abnormal EEG tracings $(n=353)$ in another study and a further $40 \%$ had mild dysrhythmias (Klinkerfuss et al., 1965). While 14 and 6 spikes may have been inaccurately interpreted as abnormal in these early studies, the controls and those with behavioral disorders should have been similarly affected. The use of EEGs in the behavioral sciences, however, has been controversial largely due to methodological flaws. With the recent advent of interest in the neurobehavioral aspects of epilepsy, it is hoped that more stringent methodological approaches will lead to greater insights (Smith et al., 1991).

The consensus is that an EEG is of value in the evaluation of ADHD only when a clear history exists of observable seizures (i.e. staring spells, absence-type behavior). Available data support the assertion that ADHD is not a form of epilepsy. Acknowledging this the EEG may still have value in the evaluation of sleep restlessness which frequently accompanies ADHD. This review explores neurophysiological abnormalities in ADHD in the context of associated sleep disturbances.

\section{SLEEP, TEMPERAMENT AND ADHD}

The contribution of sleep disturbances to the behavioral aspects of ADHD remains to be adequately defined for all subpopulations of ADHD. It is generally assumed that sleep disorders occur in ADHD yet laboratory documentation of its occurrence has remained controversial (Khan and Rechtscaffen, 1978; Kiesow and Surwillo, 1987). Sleep restlessness was a criteria for diagnosis of ADHD until the revised third edition of DSM-III (APA, 1987). Sleep disturbances may contribute to altered daytime behavior as has been observed in adults. For example adults, when deprived of rapid eye movement (REM) sleep, display overt behavior resembling children with ADHD (Luisada, 1969). Such disturbed sleep may relate to temperament considerations observed during the day.

Sleep disturbances may aggravate certain disturbances of temperament already aberrant in ADHD populations. It has been noted that boys with more active sleep patterns and difficult temperaments have brief attention spans when compared with other children $(p=0.005)$ and an

Behavioural Neurology . Vol 5 . 1992 149 
active sleep pattern is more commonly associated with infant boys. This parallels the increased incidence of ADHD in males (Weissbluth and Liu, 1983). Trommer $e t$ al. showed developmentally that children with ADHD manifest frequent arousals (32\% vs. $16 \%$ of controls) and difficulty in sleep onset (58\% vs. $23 \%$ in controls) (Trommer et al., 1982). Certain dimensions of temperament, such as sleep rhythmicity, activity, arousal, and irritability, directly relate to sleep-wake states as has been readily acknowledged in infant behavior (Keener et al., 1988). These temperament characteristics occur more often in children with night wakings (Richman, 1981).

Variability marks the nature of the sleep disturbances in ADHD children. ADHD children seem inherently more at risk from sleep disturbances because of dysregulation of their autonomic nervous sytem and variability of the degree of autonomic dysfunction may account for the lack of homogeneity of this population. Two studies have indicated an increased frequency of night wakenings in ADHD children, but no difference in total sleep time or sleep onset latency could be found (Nahas and Krynicki, 1977; Kaplan et al., 1987). The patients with ADHD $(n=25)$ had a sleep latency of $42 \mathrm{~min}$ vs. $40 \mathrm{~min}$ for those children without ADHD $(n=27)$. The effects of sleep disturbance on daytime behavior may be detectable only when sleep disturbances are extreme and thus not readily detectable, however, in earlier more subtle variations of ADHD.

\section{EPILEPTIFORM ACTIVITY, EEGs AND ADHD}

Since the advent of electroencephalography, clinicians and researchers have hoped that it would prove useful in psychiatric diagnosis. EEGs have been used to define mental disorders but the results have proved inconclusive (Jasper and Solomon, 1938; Ellingson, 1954; Daveau 1958; Werry et al., 1972). Jasper et al. found that $39 \%$ $(n=28)$ of children with behavioral problems demonstrated sudden bursts of large amplitude waves, sporadically or in regular sequence at between 2 and $4 \mathrm{~s}$, similar to interictal activity in epileptic patients (Jasper and Solomon, 1938). The authors concluded that this "definite epileptoid disorder", was responsible for their behavior problems. The authors, however, added that the group of patients should not be considered homogeneous nor were they epileptic. Further study by Jones et al. found that $12 \%$ $(n=47)$ of children with behavior disorders $(n=290)$ had focal EEG abnormalities (Jones et al., 1955). Of those 47 patients, 19 were categorized as behavior disorders without organic factors. In 1964, the concept was further elaborated and termed "subconvulsive cerebral dysrhythmia" (Gross and Wilson, 1964). Sixty-six patients with abnormal EEGs were identified from 122 children referred to a mental health clinic. Forty-five were studied and of these, $55 \%$ responded to medications, the most useful being dextroamphetamine. Anticonvulsants were also frequently used (acetazolamide, methsuximide, phenytoin and phenobarbital). The authors proposed three outcomes secondary to "subconvulsive dysrhythmia": (1) psychological dysfunction; (2) dyscontrol and distractibility; and (3) secondary psychological reactions to the first two effects (e.g. anger because the child was rejected by others following his temper outbursts). The analogy to ADHD is striking. In another study, $47 \%$ of 97 patients with behavioral disorders had abnormal EEGs compared with 9 and $19 \%$ in two control groups respectively (Stevens et al., 1968). In these children, teachers observed $47 \%, 57 \%$ and $61 \%$ as displaying hyperactivity, impulsivity or decreased attention span, respectively. In another study of 120 hyperactive children, 22 (18.5\%) were found to have EEG abnormalities (Satterfield et al., 1973a). Teachers observing these children rated the children with abnormal EEGs as more restless and fidgety. In a report of 10 children with behavioral abnormalities, five patients with ADHD had abnormal EEGs but with no recognizable epileptic seizures (Satterfield et al., 1974). A temporal or occipital spike focus was commonest. This may persist into adulthood and may explain why one-third to one-half of children with ADHD do not outgrow the need for continued methylphenidate therapy (Weiss et al., 1984).

Definitive data, however, establishing the electroencephalogram as a diagnostic aid to delineate behavioral diagnoses and treatment do not exist (Satterfield, 1973b; Rapoport et al., 1974; Schain and Reynard, 1975; Barkley, 1976; Steinhausen et al., 1984). The use of sleep laboratory studies incorporating the EEG results may however help define those patients who do not respond to methylphenidate and who may be exquisitely sensitive to the sleep altering effects of methylphenidate at a pharmacodynamic level (exceeding pharmacokinetic expectations of duration of effect based on half-life considerations), which consequently could potentiate temperament dysfunction. Perhaps the sleep of some of these children may be disrupted by mild epileptiform activity without motor manifestations. In the setting of certain EEG abnormalities, methylphenidate may not be as effective due to self-induction and furthering of these abnormalities. Sleep EEGs may therefore be useful in assessment of those patients who do not respond to conventional therapy.

\section{INTEGRATION OF EPILEPTIFORM ACTIVITY, SLEEP, TEMPERAMENT AND ADHD}

The interrelationship between epileptiform activity, sleep disturbances, and hyperactivity is pivotal to the understanding of our hypothesis. Generalized epilepsy or epileptogenic foci may cause aberrant arousal, disturbing 
sleep and result in interictal behavioral and cognitive symptoms (Dadmehr et al., 1987). Stores noted that some children with epilepsy may have a subtle disorder of sleep associated with impaired daytime behavior (Stores, 1990). Dadmehr et al. propose that patients with generalized and partial seizures may have sleep disturbances of a different character, reflecting altered cerebral excitability by afferent stimuli in the former and more localized effects of limbic or cortical hyperactivity (Dadmehr et al., 1987). In their study of 10 controls and 28 seizure patients, those with seizures had longer sleep latency and more wakefulness. More recently, Engel et al. acknowledge the controversy surrounding the relationship between hemispheric lateralizations of epileptogenic lesions and specific interictal behavioral problems, but they recognize that seizures could indirectly influence interictal behavior as a result of their effects on sleep and neuroendocrine function (Engel et al., 1991).

\section{METHYLPHENIDATE, EEGs AND ADHD}

The mainstay of ADHD therapy is psychotherapy combined with psychomotor stimulant use, mainly with methylphenidate (Dupaul and Barkley, 1990). The manufacturer of methylphenidate cautions that methylphenidate may lower seizure threshold. Additionally, methylphenidate has been associated with significant increases in latency to both sleep onset and the first REM period (Haig et al., 1974). Some contend that drug effects such as these are without significant functional effect and are not associated with pathological disruption of sleep (Haig et al., 1974). It is known, however, that disturbances may indirectly trigger a chemically stimulated stress response which in turn heightens emotionality and worsens temperament (Weissbluth, 1989). It is possible that subclinical EEG abnormalities reflect sleep disturbances or reflect a neurophysiologic process that is associated with sleep disturbance and thereby potentiate behavioral difficulties in a child with a known predisposition to ADHD. Methylphenidate may further predispose the patient to epileptiform abnormalities and may in fact counteract (leading to refractoriness to stimulant therapy as is noted in $23-27 \%$ of patients) or blunt its own therapeutic response by its effect on sleep in some patients (Barkley, 1977).

The potential of methylphenidate to predispose the patient to epileptiform activity is recognized, albeit disputed; the manufacturer purports that methylphenidate may lower the seizure threshold and should be used with great care if at all in patients with epilepsy. Recent studies, however, question this relationship. Ten children with seizures and attention deficit disorder were evaluated in terms of safety and efficacy of methylphenidate (Feldman et al., 1989). Despite manufacturer warnings that methyl- phenidate may lower the seizure threshold, they found no significant changes of epileptiform features or background activity on electroencephalograms. The authors concluded that methylphenidate is safe and effective treatment for children with seizures and concurrent attention deficit disorder. In another study, six boys with hyperactivity were compared to six normal boys. They also did not note any epileptiform activity (Haig et al., 1974). However, EEGs may show abnormal tracings in only one of four readings (i.e. $25 \%$ specificity) and it may be that these small studies may have revealed abnormalities if repeat tracings had been carried out (Goodwin and Aminoff, 1984). At present insufficient data is available to conclude firmly that methylphenidate does not affect epileptiform activity.

Use of EEGs to determine methylphenidate responsiveness in hyperactive children has also been investigated. According to a review of 36 reports involving 1400 children where prognostic indicators for methylphenidate responders were sought, EEGs are the most commonly used psychophysiological parameter (Barkley, 1977). In one study of 18 hyperkinetic children, computer-based EEG records were used with discriminant analyses to predict efficacy to stimulant therapy (Steinhausen et al., 1984). Significant differences $(p<0.05)$ in the average of the occipital and parietal channels for $\alpha$ and $\beta$ activity in the left hemisphere and for $\theta, \beta$, and $\alpha$ activity in the right hemisphere was found possibly indicating delayed maturation predicting better medication response. In a study of 57 patients Satterfield et al. found that an abnormal EEG in conjunction with an abnormal neurological examination predicted a better response to methylphenidate whereas either parameter alone was not a sufficient indicator (Satterfield et al., 1973b). A mean improvement of 55\% could be expected if both parameters were present versus $35 \%$ and $46 \%$ for those only with an abnormal neurological examination or abnormal EEG, respectively. In other investigations where the EEG was used as an index of focused arousal, methylphenidate responders were proposed as low arousal types (Hastings, 1978). The underlying premise is that hyperactive children are "underaroused" in CNS functioning and interact with their environment at a higher rate as they seek to sufficiently stimulate cortical function. Stimulant drugs provide this stimulus systemically. This hypothesis however does not explain why some individuals fail to respond to stimulant drugs. It is possible that these non-responding patients are exquisitely sensitive to the EEG-altering and sleep altering effects of methylphenidate. Other studies have been unable to validate the use of EEG findings as prognostic indicators in terms of medication response (Rapoport et al., 1974; Schain and Reynard, 1975). Markers of attention span or concentration are claimed by others to be the most useful predictors of drug response (Barkley, 1976). 


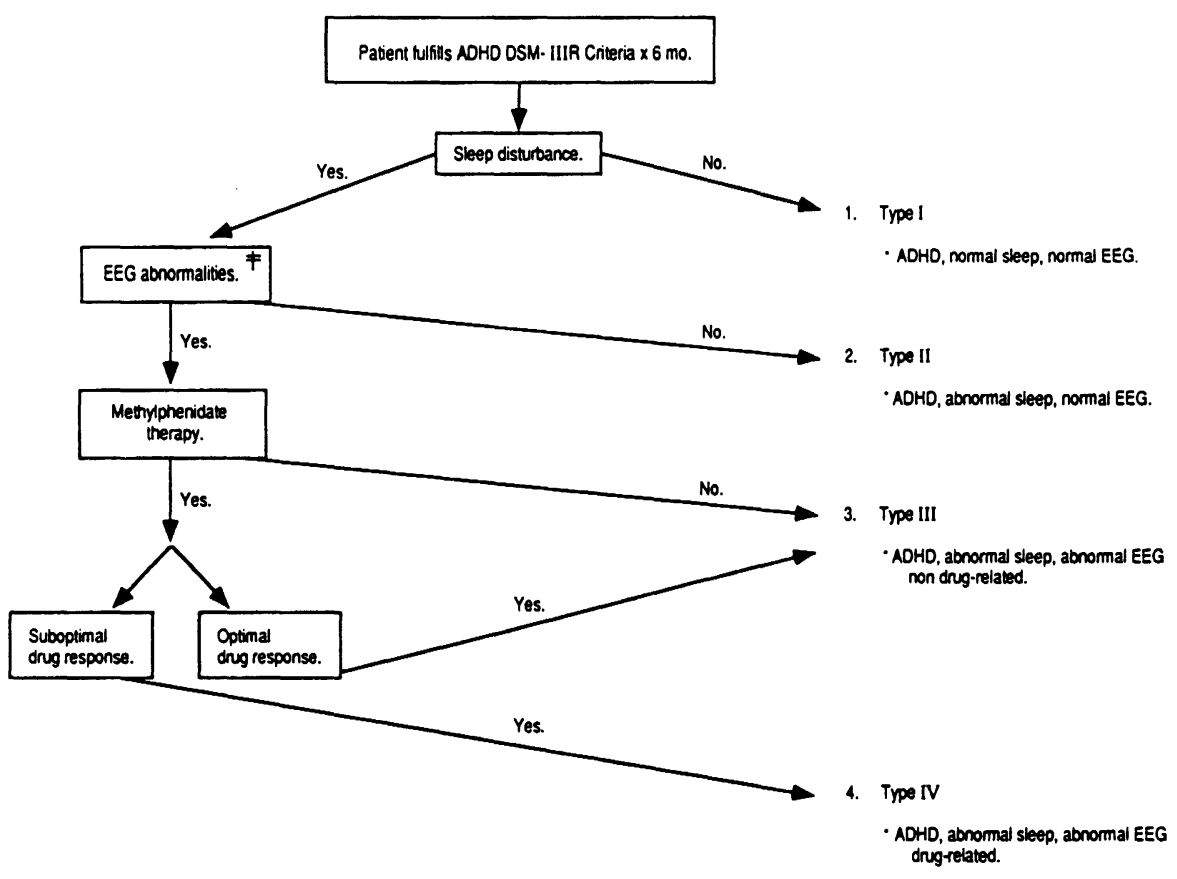

F nonepilepic

FIG. 1. Clinical subcategorizations of ADHD.

While this may be true for the majority of children with ADHD, we submit that EEGs may be useful in assessment of those who do not respond to conventional therapy.

Electroencephalograms should be used in combination with other diagnostic parameters in evaluating subpopulations of ADHD. Previously the EEG abnormalities observed in children with ADHD have been used to substantiate claims of minimal brain dysfunction. Perhaps it is now appropriate to view the abnormalities as a prognostic indicator (reflecting sleep disturbances) of response to therapy in those recalcitrant to conventional therapy or achieving suboptimal response. Characterizing the subpopulation of ADHD children who experience this epileptiform activity either prior to or coincident with methylphenidate administration has proved difficult because of the lack of homogeneity noted by Jasper.

\section{METHYLPHENIDATE, SLEEP, TEMPERAMENT AND ADHD}

The effect of anti-ADHD drugs on sleep in children with ADHD has been reported in the literature but not in the context of temperament manifestations as a consequence. Busby et al. noted significantly longer REM onset latency $(p<0.05)$ in 11 nonmedicated ADHD boys compared with 11 normal controls (Busby et al., 1981), but the authors concluded that baseline sleep is not remarkably different in ADHD children than controls and that stimulants did not significantly alter sleep patterns (Busby et al., 1981). Greenhill et al. studied nine ADHD patients both before and after 6 months of methylphenidate therapy (Greenhill et al., 1983). They found methylphenidate to be associated with delayed sleep onset, lengthened sleep and changes in certain REM variables. The drug-free ADHD children displayed no major sleep disturbances compared with control (non-ADHD) groups. Neither of these studies, however, addressed behavioral consequences of the observed sleep disturbances. Although the data obtained from the above two studies may hold true for the majority of patients conceivably a small subpopulation of ADHD may exist where subtle sleep disturbances are sufficient to evoke a behavioral response interpreted as worsening of ADHD or a poor response to methylphenidate treatment.

\section{GENERATION OF A NEW HYPOTHESIS}

Based on EEGs and sleep indices, we have formulated a hypothesis identifying four subpopulations of ADHD as shown in Fig. 1. Type I are those children with ADHD who display normal sleep patterns and EEGs. Type II ADHD patients have abnormal sleep displays but normal EEGs. Type III patients show both abnormal sleep patterns and abnormal EEGs while not clinically epileptic and the abnormalities do not appear to be drug-related whether or not they are taking methlyphenidate. The majority of 
patients with ADHD most likely will fall into Types I-III. A small subpopulation categorized Type IV have abnormal sleep patterns and abnormal EEGs which may be secondary to methylphenidate therapy. The continued sleep disturbance may relate to methylphenidate's effect on subclinical brain electrical activity leading to worsening of temperament with consequent behavior disturbances. Clinically this would be viewed as a suboptimal drug response to methylphenidate in the setting of an abnormal EEG (contrary to Satterfield et al.'s finding suggesting abnormal EEGs predict a favorable drug response).

\section{CONCLUSION}

We concur with Dahl and Puig-Antich who endorse the need for more research examining the interaction between the regulation of sleep and clinical disorders of affect and arousal in children (Dahl and Puig-Antich, 1990). It needs to be determined whether bedtime medication directed at correcting sleep patterns might raise the patient to a nearmaximal response to methylphenidate. Alternatively, adjusting the methylphenidate dosage or employing another drug without an effect on sleep or EEG activity (whether subclinical or clinically observed) may be more appropriate for a subpopulation of ADHD patients.

\section{Acknowledgement}

The authors gratefully acknowledge the thoughtful review of Dr James Frost, Director, Baylor Sleep Laboratory and Professor, Department of Neurology, Baylor College of Medicine and the assistance of Harold Wray, Department of Family Medicine, Baylor College of Medicine, in manuscript preparation.

\section{REFERENCES}

American Psychiatric Association (1987). Diagnostic and Statistical Manual of Mental Disorders, 3rd Edn, Revised (DSM III-R), pp. 50-60. APA, Washington, DC.

Barkley RA (1976) Predicting the response of hyperkinetic children to stimulant drugs: a review. Journal of Abnormal Child Psychology, 4, 327-348.

Barkley RA (1977) A review of stimulant drug research with hyperactive children. Journal of Child Psychology and Psychiatry, 18, 137-65.

Busby K, Firestone P and Pivik RT (1981) Sleep patterns in hyperkinetic and normal children. Sleep, 4, 366-383.

Dadmehr N, Congbalay DR, Pakalnis A and Drake MR Jr (1987) Sleep and waking disturbances in epilepsy. Clinical Electroencephalography, 18, 136-141.

Dahl RE and Puig Antich J (1990) Sleep disturbances in child and adolescent psychiatric disorders. Pediatrician, 17, 32-37.

Daveau M (1958) The EEG of 150 children with behavior disorders. Electroencephalography and Clinical Neurophysiology, 10, 198.

Dupaul GJ and Barkley RA. Medication therapy. In: Attention Deficit Hyperactivity Disorder (Ed. RA Barkley), pp. 573612. Guilford Press, New York.
Ellingson RJ (1954) The incidence of EEG abnormality among patients with mental disorders of apparently nonorganic origin: a critical review. American Journal of Psychiatry, 111, 263-275.

Engel J Jr, Bandler R, Griffith NC and Caldecott Hazard S (1991) Neurobiological evidence for epilepsy-induced interictal disturbances. Advances in Neurology, 55, 97-111.

Feldman H, Crumrine P, Handen BL, Alvin R and Teodori J (1989) Methylphenidate in children with seizures and attention deficit disorder. American Journal of Diseases of Children, 143, 1081-1086.

Goodin DS and Aminoff MF (1984) Does the interictal EEG have a role in the diagnosis of epilepsy? The Lancet, $\mathbf{1}$, 837-839.

Greenhill L, Puig-Antich, J, Goetz R, Hanlon C and Davies M (1983) Sleep architecture and REM sleep measures in prepubertal children with attention deficit disorder with hyperactivity. Sleep, 6, 91-101.

Gross MD and Wilson WC (1964) Behavior disorders of children with cerebral dysrhythmias: successful treatment of subconvulsive dysrhythmia with anticonvulsants. Archives of General Psychiatry, 11, 610-619.

Haigh JR, Schroeder CS and Schroeder SR (1974) Effects of methylphenidate on hyperactive children's sleep. Psychopharmacology, 37, 185-188.

Hastings JE (1978) A review of psychophysiological research with hyperkinetic children. Journal of Abnormal Child Psychology, 6, 413-447.

Jasper HH and Solomon P (1938) Electroencephalographic analyses of behavior problem children. American Journal of Psychiatry, 95, 641-658.

Jones E, Bachi BK and Waggoner RW (1955) Focal abnormalities of electroencephalogram in juveniles with behavior disorders. Journal of Nervous and Mental Disease, 122, 28-35.

Kaplan BJ, McNicol J, Conte RA and Moghadam HK (1987) Sleep disturbance in preschool-aged hyperactive and nonhyperactive children. Pediatrics, 80, 839-844.

Keener MA, Zeanah CH and Anders TF (1988) Infant temperament, sleep organization, and night-time parental interventions. Pediatrics, 81, 762-771.

Khan A and Rechtscaffen A (1978) Sleep patterns and sleep spindles in hyperkientic children. Sleep Research, 7, 137.

Kiesow NA and Surwillo WW (1987) Sleep spindles in the EEGs of Hyperactive children. Psychological Reports, 60, 139-144.

Klinkerfuss GH, Lange PH, Weinberg WA and O'Leary JL (1965) Electroencephalographic abnormalities of children with hyperkinetic behavior. Neurology, 15, 883-891.

Lahey BB, Piacentini JC, McBurnett K, Stone P, Hartdagan S and Hynd G (1988) Psychopathology in the parents of children with conduct disorder and hyperactivity. Journal of the American Academy of Child Adolescent Psychiatry, 27, 163-170.

Luisada PV (1969) REM deprivation and hyperactivity in children. Chicago Medical School Quarterly, 28, 97-108.

Nahas AD and Krynicki V (1977) Effect of methylphenidate on sleep stages and ultradian rhythms in hyperactive children. Journal of Nervous Mental Disease, 164, 66-69.

Rapoport JL, Quinn PO, Bradbard G, Riddle D and Brooks E (1974) Imipramine and methylphenidate treatments of hyperactive boys. Archives of General Psychiatry, 30, 789-793.

Richman N (1981) Sleep problems in young children. Archives of Disease in Childhood, 56, 491-493.

Satterfield JH, Cantwell DP, Saul RE, Lesser LI and Podosin RL (1973a) Response to stimulant drug treatment in hyperactive 
children: prediction from EEG and neurological findings. Journal of Autism and Child Schizophrenia, 3, 36-48.

Satterfield JH, Lesser LI, Saul RE and Cantwell DP (1973b) EEG aspects in the diagnosis and treatment of minimal brain dysfunction. Annals of the New York Academy of Sciences, 205, 274-282.

Satterfield JH, Cantwell DP, Saul RE and Yusin A (1974) Intelligence, academic achievement and EEG and abnormalities in hyperactive children. American Journal of Psychiatry, 131, 391-395.

Schain RJ and Reynard CL (1975) Observations on effects of a central stimulant drug (methylphenidate) in children with hyperactive behavior. Pediatrics, 55, 709-716.

Smith DB, Treiman DM and Trimble MR (Eds.) (1991) Neurobehavioral problems in epilepsy. In: Advances in Neurology. Raven Press, New York.

Steinhausen HC, Roman G and Gobel D (1984) Computer analyzed EEG in methylphenidate-responsive hyperactive children. Neuropediatrics, 15, 28-32.

Stevens JR, Sachdeve K and Milstein V (1968) Behavior disorders of childhood and the electroencephalogram. Archives of Neurology, 18, 160-177.

Stores G (1990) Electroencephalographic parameters in assessing the cognitive function of children with epilepsy. Epilepsia,
31(Suppl. 4), S45-S49.

Trommer BL, Hoeppner JB, Rosenberg RS, Armstrong KJ and Rothstein JA (1988) Sleep disturbance in children with attention deficit disorder. Annals of Neurology, 24, 322.

Walker EF and Katy Kl (1958) Brain damaged children. California Medicine 88, 320-324.

Walker WG (1950) Clinical aspects of maturation studies and normal variations. In: Electroencephalography (Eds. D Hill and G Parr), pp. 214-218. MacDonald, London.

Weiss G, Hechtman L, Milroy T and Perlman T (1984) Psychiatric status of hyperactives as adults. A controlled prospective 15-year follow-up of 63 hyperactive children. Journal of the American Academy of Child Psychiatry, 24, 211-220.

Weissbluth M and Liu K (1983) Sleep patterns, attention span, and infant temperament. Developments in Behavioural Pediatrics, 4, 34-36.

Weissbluth M (1989) Sleep-temperament interactions. In: Clinical and Educational Applications of Temperament Research (Eds WB Carey and SC McDevitt), pp. 113-116. Swets North American, Berwyn, PA.

Werry JS, Minde K, Gukzman A, Weiss G, Dogan K and Hoy E (1972) Studies on the hyperactive child; VII: neurological status compared with neurotic and normal children. American Journal of Orthopsychiatry, 42, 441-450. 


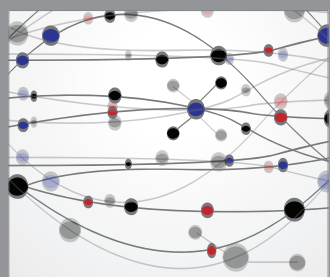

The Scientific World Journal
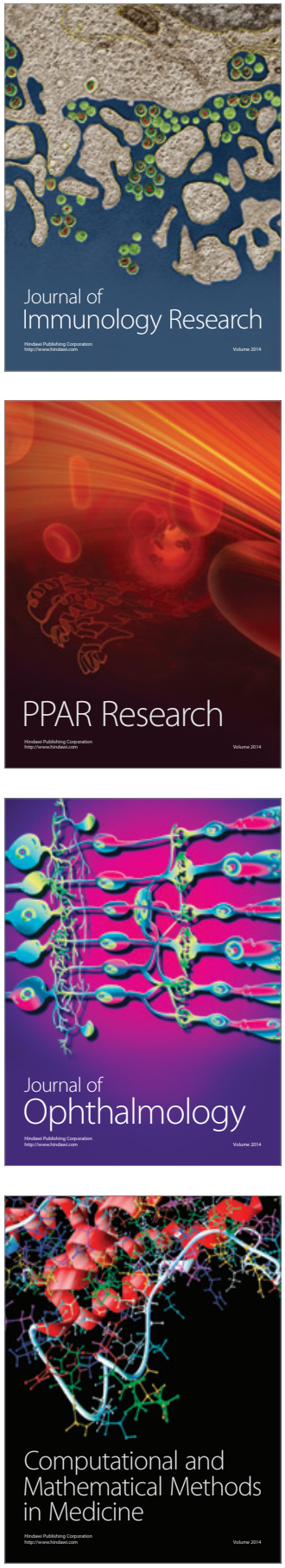

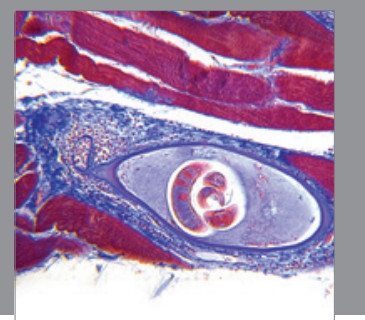

Gastroenterology

Research and Practice
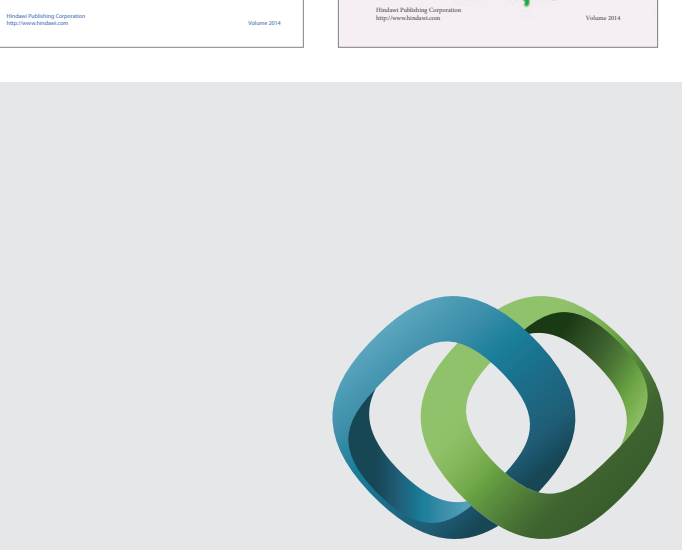

\section{Hindawi}

Submit your manuscripts at

http://www.hindawi.com
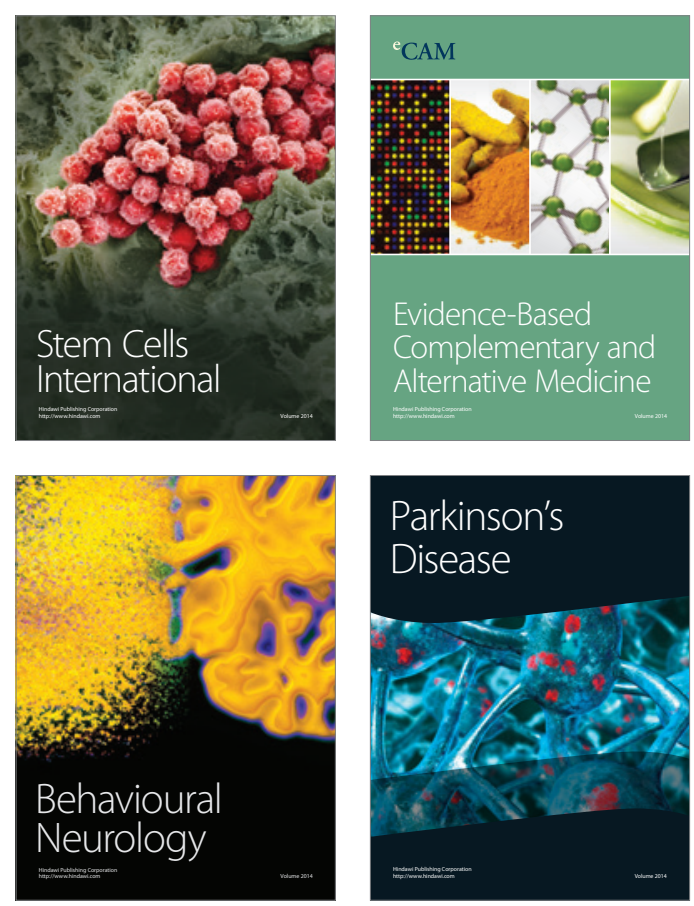

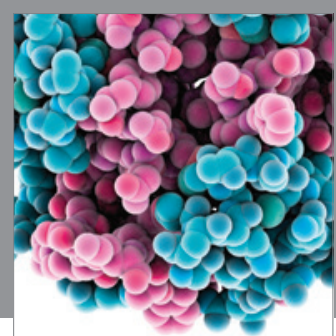

Journal of
Diabetes Research

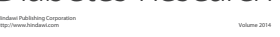

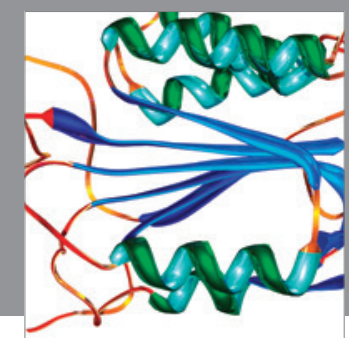

Disease Markers
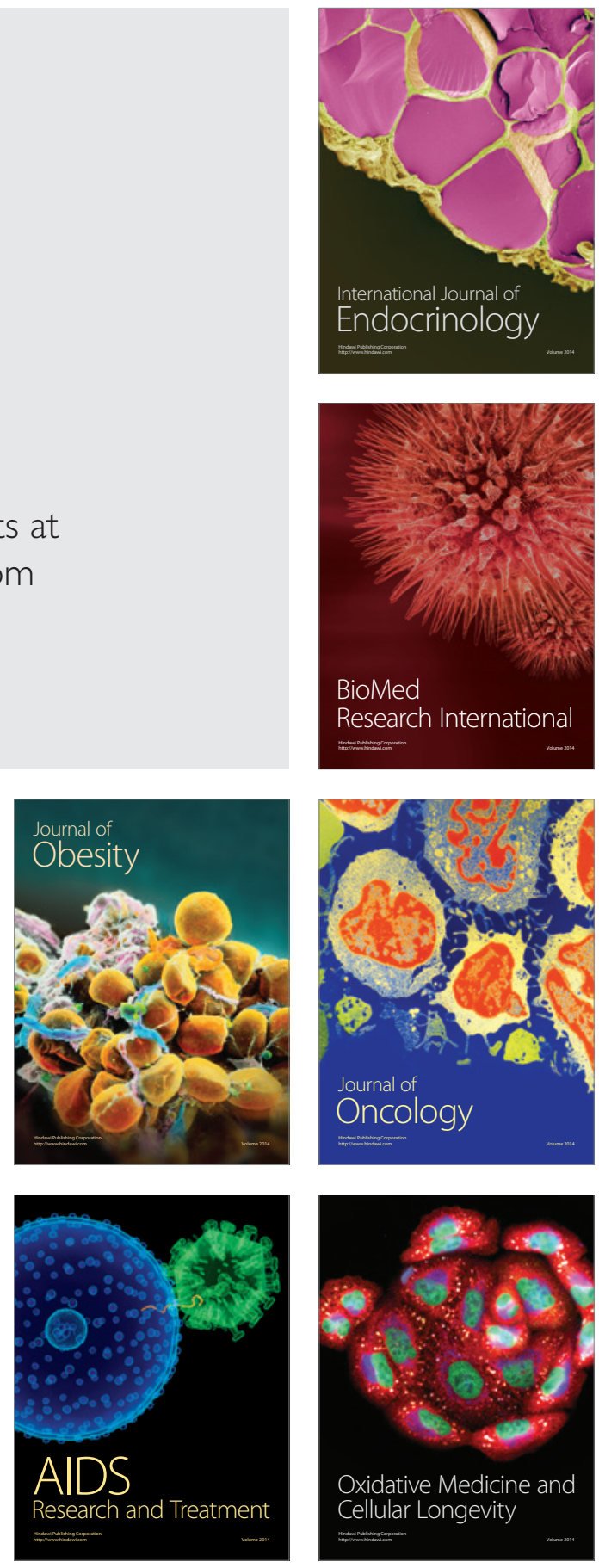